\title{
Revisión sistemática de la literatura sobre el fenómeno del techo de cristal en las universidades españolas
}

Nazareth Gallego-Morón, Mauricio A. Matus-López y Lina Gálvez-Muñoz

\section{RESUMEN}

En el presente trabajo se realiza una revisión sistemática de la literatura sobre el fenómeno del techo de cristal en las universidades españolas. Esta tuvo un horizonte temporal de diez años e incluyó los estudios publicados en las principales bases de datos de revistas indexadas y de organismos oficiales. Se detectan tres características de los estudios: 1) escasez de investigaciones empíricas, 2) estudios de casos aislados y 3) falta de consenso sobre las causas del fenómeno. Se concluye la necesidad de realizar estudios empíricos a nivel nacional centrados en el análisis de las barreras organizacionales.

Palabras clave: estudios de género, discriminación sexual, educación superior, estado del arte, universidades, España.

\section{Nazareth Gallego-Morón}

\section{Mauricio A. Matus-López}




\section{Revisão sistemática da literatura sobre o fenómeno do teto de cristal nas universidades espanholas}

\section{RESUMO}

No presente trabalho se realiza uma revisão sistemática da literatura sobre o fenómeno do teto de cristal nas universidades espanholas. Esta teve um horizonte temporal de dez anos e incluiu os estudos publicados nas principais bases de dados de revistas indexadas e de organismos oficiais. Se detectam três características dos estudos: 1) escassez de pesquisas empíricas, 2) estudos de casos isolados e 3) falta de consenso sobre as causas do fenómeno. Se conclui a necessidade de realizar estudos empíricos a nível nacional centrados na análise das barreiras organizacionais.

Palavras chave: estudos de gênero, discriminação sexual, educação superior, estado da arte, universidades, Espanha .

\section{Systematic review of the literature on the glass ceiling phenomenon in Spanish universities}

\section{ABSTRACT}

In the present article the authors carry out a systematic review of the literature on the phenomenon of the glass ceiling in Spanish universities. The analyzed period interval is a ten-year period and included the studies published in the main databases of indexed journals and official bodies. Three features of the analyzed studies are detected: 1) scarcity of empirical research, 2) studies of isolated cases and 3) lack of consensus on the causes of the phenomenon. The authors come to the conclusion that it is necessary to carry out for empirical studies at national level focused on the analysis of organizational barriers.

Key words: gender studies, sexual discrimination, higher education, state of the art, universities, Spain. 


\section{Introducción}

Más de un siglo ha pasado desde que en 1910 las mujeres pudieran acceder a la educación universitaria en España (Flecha, 2010). Desde entonces se han incorporado rápidamente al alumnado y a la plantilla del profesorado. Hace dos décadas, en 1999, eran el 32.5\% del personal docente e investigador (PDI) (INE, 1999). En la actualidad, se produce una presencia equilibrada, atendiendo a la horquilla de representación proporcional de ambos sexos del 40$60 \%$. Sin embargo, pese a este avance, su participación es menor a medida que se avanza en la carrera académica. Según datos del Ministerio de Educación, Cultura y Deporte (MECD, 2018), para los centros propios de las universidades públicas españolas, en el curso académico 2016-2017 las mujeres eran mayoría en el alumnado de grado (54.5\%), así como en los masters $(52.3 \%)$, y prácticamente iguales en los cursos de doctorado (49.8\%) y mayoría en las tesis doctorales aprobadas en $2016(50.8 \%)$. No obstante, en el PDI representaban el 40.1\%, mientras que en las cátedras de universidad la participación descendía hasta el 21.3\% (MECD, 2018).

Esta situación se replica a nivel internacional, así, en 2016, en cuatro de cada cinco países con datos disponibles, el porcentaje de mujeres egresadas en educación superior era mayor que el de varones. No obstante, éstas representaban únicamente el $29 \%$ del total del personal investigador. La región de América Latina y el Caribe se presenta como una excepción, con un $45 \%$ de investigadoras (UIS, 2019). Si se atiende a la participación femenina en las altas categorías académicas (Grado A), ésta disminuye considerablemente. A modo de ejemplo, en 2016 éstas constituían: el 24\% en la Unión Europea (UE-28) (European Commission, 2019), 27\% en los Estados Unidos (NCES, 2017), 25.8\% en la India y 23.7\% en Japón (Catalyst, 2017). Se trata del fenómeno del techo de cristal, el proceso por el que las mujeres no alcanzan las categorías más altas de la pirámide organizacional. Un entramado de barreras y obstáculos que perjudica más a ellas que a ellos (Morrison et al., 1987) y que se da a nivel mundial, incluso en aquellos contextos con índices de igualdad superiores, como es el caso de los países nórdicos (European Commission, 2019). Como Adler (1986: 18) señala, "en todos los países, occidentales y orientales; comunistas, socialistas y capitalistas; desarrollados y en vía de desarrollo; la proporción de mujeres en puestos de liderazgo es menor que el de los hombres, viéndose reducida a medida que se avanza en la pirámide organizacional". Además, la escasa presencia femenina en los puestos de poder se produce en todos los ámbitos, públicos y privados, como se muestra en el informe "The World's Women 2015: Trends and Statistics" (United Nations, 2015).

El interés científico por analizar las características, causas y efectos del techo de cristal en la universidad es amplio. Son múltiples los estudios realizados y variadas las metodologías empleadas, como las investigaciones realizadas por Gewinner (2016) y O'Connor et al. (2017) para el contexto europeo; Probert (2005) para Australia; Peterson (2016) para Estados Unidos; o Wesarat y Mathew (2017) para el contexto asiático, y otros tantos en países de América Latina (Méndez y Rojas, 2014; Rebolledo y Espinosa, 2016). Sus resultados reflejan el carácter estructural y la transversalidad del fenómeno. Es decir, la intervención de barreras de diferentes tipologías, que obstaculizan las trayectorias de las académicas.

En España, los primeros estudios se remontan a casi 30 años, cuando Fernández (1989) cuantificó la participación de las mujeres en la universidad española. No obstante, es hasta fines de la década de los años noventa y principios del nuevo milenio, cuando se realizaron estudios específicos sobre la participación de las mujeres en la cátedra. Pilares de este estudio fueron los trabajos de García de León y García de Cortázar (1997, 2001), que avanzaron desde la descripción hacia las causas del fenómeno. 
Lamentablemente no existió continuidad a estos estudios y su abordaje fue esporádico durante los años siguientes. Hubo que esperar hasta la última década para ver surgir nuevamente investigaciones sobre esta problemática. Diversos factores, como la mayor sensibilidad hacia la discriminación de género o la aplicación de nuevas metodologías, llevó a que, en los últimos diez años, se publicaran casi medio centenar de investigaciones.

La existencia del techo de cristal en las universidades repercute de un modo negativo en las trayectorias de las académicas. Así, las mujeres ven reducido su tiempo y disponibilidad, debido, sobre todo, a las dificultades de conciliación (Antón, 2005) y ausencia de corresponsabilidad masculina (Ion et al., 2013), viendo afectados sus niveles de productividad y, por tanto, sus posibilidades de promoción (Bozzon et al., 2017). Junto a las dificultades familiares, la existencia de obstáculos organizacionales y sociales, como las redes informales de poder y los estereotipos y roles de género juegan un papel fundamental (Guil et al., 2005). Esta situación de desigualdad tiene consecuencias no sólo en la vida personal y profesional de las mujeres, para las organizaciones el coste de no luchar contra la discriminación de género en el mercado laboral se traduce en la pérdida potencial de capital humano con altos niveles de instrucción y capacitación. Así, en términos económicos, se estima que el costo de la subutilización del capital humano femenino para la UE-28 supera los 370 mil millones de euros, es decir, el 2.8\% del PIB (Eurofound, 2016: 37).

En este contexto, el objetivo del presente estudio consiste en analizar la evidencia científica sobre este fenómeno en las universidades españolas en la última década. Para ello se realizó una revisión sistemática de la literatura entre 2005 y 2016. Los resultados permitieron abordar tres ejes de análisis: concepto de techo de cristal, metodologías de investigación y resultados sobre las causas. La novedad reside en la ausencia de investigaciones previas que se ocupen en revisar de manera exhaustiva y sistemática el estado de la cuestión en España, siendo analizados desde una perspectiva internacional. Éste ofrece la oportunidad de obtener una panorámica de lo que se sabe y lo que se ignora sobre este fenómeno, qué y cómo se ha investigado, y cuáles han sido los principales resultados. El fin último es doble: por un lado, poner de relieve los desafíos a los que la investigación debe hacer frente en el estudio del fenómeno del techo de cristal en la academia, por otro lado, se constituye como diagnóstico necesario para el diseño e implementación de medidas de intervención por parte de organismos públicos y de las propias universidades.

El presente trabajo se estructura de la siguiente forma. En el segundo apartado se desarrollan las cuestiones metodológicas referentes a la selección de fuentes, los criterios de inclusión y exclusión adoptados y la clasificación de los resultados realizada. En tercer lugar, se presentan los resultados obtenidos tras la revisión y análisis de la literatura seleccionada. Para ello, se han diferenciado distintos subapartados donde: 1) se describen aquellos artículos que definen el concepto de techo de cristal, 2) se desarrolla la literatura que mide cuantitativamente el fenómeno, 3) se analiza el diseño metodológico de los estudios y 4) se identifican las barreras y obstáculos que son señalados y que las mujeres deben superar para promocionar. Finalmente, y a modo de conclusión, se presentan desde un punto de vista constructivo algunas cuestiones sobre las que es necesario profundizar y dirigir posibles futuras líneas de investigación en esta materia.

\section{Metodología}

Se realizó una investigación secundaria a partir de documentos primarios, consistente en la revisión de los estudios e investigaciones sobre el techo de cristal en las universidades españolas, publicados en el periodo 2005-2016 (tabla 1). Se procuró realizar la revisión de la manera más sistemática y rigurosa posible, evitando sesgos subjetivos. 
Tabla 1. Descripción de la revisión sistemática de la literatura

\begin{tabular}{|l|l|}
\hline Fuentes consultadas & $\begin{array}{l}\text { Web of Science, Scopus, EBSCOThost (PsycINFO), ProQuest (Social Science Data Base), ISOC (CSIC), Dialnet, Redined y Rebiun. Además, } \\
\text { los informes oficiales de MECD, Consejo de Rectores e Instituto de la Mujer. }\end{array}$ \\
\hline Periodo & Estudios publicados entre 2005 y 2016. \\
\hline $\begin{array}{l}\text { Descriptores (en } \\
\text { español y en inglés) }\end{array}$ & $\begin{array}{l}\text { Universidad AND Género AND Discriminación (Universit* AND Gender AND Discrimination) } \\
\text { Educación Superior AND Género AND Discriminación (Higher Education AND Gender AND Discrimination) } \\
\text { Universidad OR Educación Superior AND Techo de Cristal (Universit* OR Higher Education AND Glass Ceiling) } \\
\text { Universidad OR Educación Superior AND Segregación Vertical (Universit* OR Higher Education AND vertical segregation) }\end{array}$ \\
\hline Complementariedad & Revisión completa de la literatura citada por los/as autores/as de los documentos identificados. \\
\hline $\begin{array}{l}\text { Criterios de } \\
\text { inclusión }\end{array}$ & $\begin{array}{l}\text { Que el sujeto de estudio sea el personal docente e investigador universitario; que el objetivo del estudio tenga relación directa con la } \\
\text { carrera académica; que se contextualice en la universidad española; que aborde las diferencias entre mujeres y hombres. }\end{array}$ \\
\hline Revisión & La revisión y análisis de cada uno de los textos fue realizado por los autores, y en caso de duda se consultó la opinión de otra experta. \\
\hline Periodo de análisis & Septiembre de 2016 - enero de 2018. \\
\hline
\end{tabular}

\section{Selección de fuentes}

Se revisaron las bases internacionales Web of Science, Scopus, EBSCOThost (PsycINFO) y ProQuest (Social Science Data Base); y las bases nacionales ISOC (CSIC), Dialnet, Redined y Rebiun, además de las publicaciones oficiales españolas del Ministerio de Educación, Cultura y Deporte (MECD), del Consejo de Rectores y del Instituto de la Mujer. El periodo de publicación se restringió de enero de 2005 a agosto de 2016.

Las palabras género y mujer, utilizadas como descriptores, pueden generar una gran cantidad de información que no se encuentra relacionada con nuestro campo de estudio. Para que fuera lo más productiva posible, las siguientes palabras claves han sido combinadas en diversas ecuaciones de búsqueda, tanto en español como en inglés: universidad (española), educación superior, techo de cristal, segregación, vertical, discriminación, género y mujer.

El diseño de una sola ecuación compleja que agrupara las palabras anteriores no permitía una búsqueda fructífera que arrojara los resultados adecuados. En ocasiones, éstos eran inexistentes. Esta situación se deriva de la diversidad en sus características de las bases de datos consultadas (opciones de búsqueda posibles, origen de la base de datos, calidad, etcétera). Por este motivo, se aplicó un algoritmo de búsqueda complejo para cada base citada.

De manera complementaria a este proceso, se procedió a la revisión total de la literatura creada por los/as autores/as de los documentos seleccionados en las bases de datos consultadas. Además, los textos más citados e influyentes conducen a otros textos y a la revisión de referencias cruzadas a medida que se procedía a la revisión de los artículos seleccionados, lo que amplió la selección de documentos objetos de análisis de este estudio.

\section{Criterios de inclusión y exclusión}

Los criterios de inclusión fueron: 1) que el sujeto de estudio sea el personal docente e investigador universitario, 2) que el objetivo del estudio tenga relación directa con la carrera académica, 3) que se contextualice en la universidad española y 4) que aborde las diferencias entre mujeres y hombres.

La revisión de los criterios fue realizada artículo a artículo por los/as autores/as, con consultas a expertos/as en caso de desacuerdo. El resultado fue la selección de 46 documentos sometidos a análisis (tabla 2), siendo excluidos todos aquellos trabajos que no cumplieran los criterios de inclusión mencionados anteriormente. 
Tabla 2. Listado de documentos revisados y analizados

\begin{tabular}{|c|c|c|c|c|c|c|c|c|}
\hline \multirow{2}{*}{ Categoría } & \multirow{2}{*}{ Def* } & \multirow{2}{*}{ Med $^{* *}$} & \multicolumn{5}{|c|}{ Estudios empíricos Métodos de investigación utilizados ${ }^{* * *}$} & \multirow{2}{*}{$\begin{array}{c}\text { Analizan } \\
\text { las } \\
\text { causas }\end{array}$} \\
\hline & & & Ent & Enc & $G D$ & $H d V$ & $O D$ & \\
\hline Arcos et al. (2013) & - & PDI & & & & & & \\
\hline Antón (2005) & - & A & & $x$ & & & & $x$ \\
\hline Blahopoulou et al. (2011) & - & A & & & & & & \\
\hline Cáceres et al. (2015) & - & - & & & & & & \\
\hline Cinta y Velázquez (2013) & O & - & & & & $x$ & $x$ & \\
\hline De Filippo et al. (2009) & O & PDI & & & & & & $x$ \\
\hline Díaz y Cuevas (2015) & O & G & & & & & & \\
\hline Durán e lon (2014) & - & - & $x$ & & & & & \\
\hline Escolano (2006) & $P$ & PDI & $x$ & $x$ & & & & $x$ \\
\hline García (2010) & - & A & & & & & & $x$ \\
\hline García de León et al. (2010) & - & PDI & $x$ & & & & & \\
\hline Guil (2005a) & - & PDI & & & & & & \\
\hline Guil (2005b) & $P$ & A & & & $x$ & & & $x$ \\
\hline Guil (2007) & P & A & & & & & & \\
\hline Guil (2008) & $\mathrm{P}$ & PDI & & & & & & \\
\hline Guil et al. (2005) & P & A & $x$ & $x$ & $x$ & & & $x$ \\
\hline Guil y Vila (2006) & - & PDI & & & & & & \\
\hline lon et al.(2013) & - & - & $x$ & & & & & $x$ \\
\hline López et al. (2014) & - & A & & & & & & \\
\hline Lorenzo et al. (2007) & - & G & & & & & & \\
\hline Lozano e Iglesias (2014) & - & PDI & & & & & & \\
\hline Lozano et al. (2014) & - & A & $x$ & & & & & \\
\hline Martínez et al. (2010) & - & A & & & & & & \\
\hline Martínez et al. (2011) & P & A & & & $x$ & & & $x$ \\
\hline Matus-López y Gallego-Moron (2015) & O & PDI & & $x$ & & & & \\
\hline Montané y Pessoa (2012) & - & PDI & & & & & & \\
\hline Morales et al. (2010) & $\mathrm{O}$ & A & $x$ & & & & & \\
\hline
\end{tabular}




\begin{tabular}{|c|c|c|c|c|c|c|c|}
\hline Pastor et al. (2010) & - & PDI & & $x$ & & & $\mathrm{x}$ \\
\hline Pérez et al. (2005) & - & PDI & & & & & \\
\hline Poy (2009) & - & PDI & & & & & \\
\hline Puy et al. (2007) & - & A & $x$ & $x$ & & & $\mathrm{x}$ \\
\hline Rodríguez (2006) & - & A & & & & & \\
\hline Roldán et al. (2012) & A & A & & & & & \\
\hline Sales (2009) & - & A & & & & & \\
\hline Sánchez et al. (2011) & - & PDI & & & & & $\mathrm{x}$ \\
\hline Simelio y Rovetto (2008a) & - & PDI & $x$ & & & & $\mathrm{x}$ \\
\hline Simelio y Rovetto (2008b) & - & PDI & $x$ & & & & $\mathrm{x}$ \\
\hline Soleto y Oubiña (2005) & - & PDI & & & & & $\mathrm{x}$ \\
\hline Tomás y Durán (2009) & - & - & & & $x$ & $x$ & \\
\hline Tomás y Guillamón (2009) & A & - & $x$ & $x$ & & & $\mathrm{x}$ \\
\hline Tomás et al. (2008) & - & - & $x$ & $x$ & & & $\mathrm{x}$ \\
\hline Tomás et al. (2010) & - & - & $x$ & $x$ & & & $x$ \\
\hline Tomás et al. (2012) & - & - & $x$ & & & & \\
\hline Torres y Pau (2012) & $P$ & PDI & & & & & \\
\hline Vázquez y Elston (2006) & - & PDI & $x$ & & & & $\mathrm{x}$ \\
\hline Vega y Santos (2010) & - & A & & & & & \\
\hline
\end{tabular}

* Definición de otro/a autor/a (O), propia (P) o ambas (A).

** Personal docente e investigador (PDI), cargos de gobierno y gestión (G) o ambos (A).

*** Entrevista (Ent), cuestionario o encuesta (Enc), grupo de discusión (GD), historia de vida (HdV) y observación participante (OP).

\section{Clasificación de los resultados}

Para cada uno de los documentos seleccionados se realizó un mapa conceptual en formato ficha donde se incluyen las características principales de los textos analizados, objetivos, métodos utilizados y los principales resultados. La revisión y análisis de cada uno de los textos fue realizado por los/as autores/as de este trabajo, y en caso de duda se buscó la opinión y consenso con terceras personas expertas en la materia.

La clasificación se realizó de forma no excluyente en las siguientes categorías: a) documentos que delimitan el fenómeno y/o sus características, b) documentos que miden cuantitativamente el techo de cristal y c) documentos que analizan las causas y proponen una o más explicaciones del fenómeno.

\section{Resultados}

El primer y segundo resultado fue la descripción y medición del concepto de techo de cristal en su contexto universitario, respectivamente. El tercer producto consistió en el análisis de las metodologías utilizadas en las investigaciones. Por último, se identificaron las causas que explican la menor proporción de mujeres en las categorías académicas más altas. 


\section{Comprendiendo el concepto de techo de cristal}

Aunque parezca una cuestión dada, no todos los estudios definen en qué consiste el techo de cristal. De hecho, sólo una tercera parte de los trabajos analizados entrega una definición explícita del concepto, ya sea propia o citada de otro/a autor/a.

Entre las definiciones citadas, las más utilizadas son las de Morrison et al. (1987), Barberá et al. (2001), Mingorance et al. (2004) y Tomás y Guillamón (2009). La definición de Morrison et al. (1987) es posiblemente la más conocida y utilizada, incluso fuera del ámbito académico. Es una conceptualización originaria, pero que ha ido sufriendo cambios con el tiempo. Para estos/as autores/as, el techo de cristal es una barrera que se interpone en la parte alta de la carrera laboral de las mujeres, y que se caracteriza por ser invisible, acotada y sólida.

Los otros tres trabajos más citados ponen de relieve la relativización de la solidez de la barrera. Es decir, postulan que esta sí puede ser rota, o quebrada, y que, aunque son pocas, la evidencia confirma que hay mujeres que logran alcanzar las posiciones más altas de las categorías profesionales. No obstante, no es una opinión de total consenso. Para Martínez et al. (2011), ésta es tan infranqueable, que más bien cabe hablar de un "techo de cemento" en lugar de un techo de cristal.

Asimismo, Guil et al. (2005) critican la condición de invisibilidad del fenómeno. Para estas autoras, su existencia es visible a través de las cifras de personal y de las quejas de las mujeres que, considerando tener los méritos suficientes, no logran promocionar. Es decir, su invisibilidad no es tal, sino que lo que ocurre es que la problemática no está presente en la discusión pública.

A estos matices e interpretaciones se agregan otros tres. En primer lugar, el impacto de la cuestión histórico/temporal. Guil (2005b, 2007, 2008) y Martínez et al. (2011) llaman la atención acerca de la pérdida de importancia de la baja participación laboral histórica de las mujeres. En 1976 sólo el 28.4\% de la población ocupada en España eran mujeres. En 2016 este porcentaje había aumentado hasta el 45.5\% (INE, 2016). Así, el techo de cristal se perfila hoy en mayor relación a los frenos en la promoción de una cualificada y meritoria cantidad de profesionales mujeres, y no sólo a una baja proporción en el total poblacional.

En segundo lugar, se pone en juicio la delimitación del fenómeno. Es decir, no existiría una única pared sino un entramadado de obstáculos invisibles que frenan el ascenso de las mujeres (Roldán et al., 2012). Este entramado es para otros/as un "laberinto de cristal", que refleja la existencia de múltiples caminos o series de decisiones sobre la carrera académica que no llevan a la cima de la pirámide organizacional (Matus-López y Gallego-Morón, 2015).

Como se puede observar, la descripción del techo de cristal parece algo intuitivo. Una barrera de acceso a una posición superior en la escala laboral. Caracterizada como "de cristal", porque es posible ver a través de ella a quienes la han cruzado, pero que, por distintos motivos, ajenos a los méritos, algunas personas, mayoritariamente mujeres, no logran superarla. Sin embargo, a la hora de estudiar de forma más profunda el fenómeno, se va desplegando una visión compleja de la problemática, y queda en evidencia la concepción simplificada de la concepción intuitiva. De este modo, el techo de cristal no es una única barrera y su naturaleza está relacionada con otras discriminaciones más sutiles, o menos pronunciadas, que afectan a todo el recorrido laboral. Determinar las características propias y específicas del fenómeno no parece fácil, y de ahí que, como se analiza en este artículo, los avances en la identificación de las causas sean limitados.

\section{Midiendo el techo de cristal en la academia española}

Cuatro de cada cinco estudios analizados cuantifican, de alguna manera, el techo de cristal en las 
universidades españolas, y con base en ellos, es posible identificar dos enfoques. Uno por puestos de responsabilidad y otro por categoría académica.

\section{El acceso al poder}

Este enfoque busca cuantificar cuántas mujeres, proporcionalmente y en términos absolutos, acceden a puestos de responsabilidad académica. Cuando se aborda este enfoque suele ser complementario al enfoque de categoría académica. Es decir, no se plantea como un objetivo específico, sino como parte de la descripción de la presencia de las mujeres en las universidades. Sólo en dos casos los trabajos se centran únicamente en esta problemática (Díaz y Guevas, 2015; Lorenzo et al., 2007). El análisis del techo de cristal, desde esta perspectiva, pasa por definir qué es un puesto de poder y valorar cómo se accede a él. Aunque existe cierto consenso en que los puestos de poder están acotados, al menos, a los órganos de gobierno del rectorado, recanatos, dirección de centros y departamentos, el sistema de nominación no es homogéneo. En algunos casos son resultado de decisiones unipersonales de otros cargos y, en otros, votaciones de los miembros de la universidad. Otra complicación de estos trabajos es que requiere la recolección de información actualizada de quién ocupa los puestos de poder. No existe un registro nacional de ellos y su búsqueda se realiza universidad a universidad y centro a centro, lo que complica el seguimiento.

Pese a estas limitaciones, es posible identificar una dimensión del techo de cristal como la diferencia de hombres y mujeres en acceso a los puestos de poder en el ámbito académico. De este modo, Díaz y Cuevas (2015) analizaron la composición por sexo de los equipos rectorales del total de las 50 universidades públicas españolas. Encontraron que en 2015 las mujeres dirigían únicamente el 2\% del total de rectorados y el 38.3\% de vicerrectorados. En el pasado curso académico 2016/2017, el porcentaje de representación femenina en los rectorados había aumentado hasta el 8\%, lo que en términos absolutos se traduce en cuatro rectoras, en concreto, en la Universidad Autónoma de Barcelona, y en las universidades de Granada, Huelva y País Vasco (CRUE, 2018).

\section{El acceso a la cátedra}

Es el enfoque más utilizado y puede o no considerar la valoración del acceso a los puestos de poder. Consiste en una medición enfocada a determinar la diferencia entre mujeres y hombres en la/s categoría/s más alta/s de la academia española, principalmente la cátedra.

Es la más utilizada porque los datos de personal docente y de investigación (PDI) están disponibles, para el total nacional y por universidad, en la Estadística de Personal de las Universidades del Ministerio de Educación (MECD, 2018) (tabla 3). Además, los estudios citan los informes oficiales de la Unión Europea, del Instituto Nacional de Estadística (INE), del Instituto de la Mujer, CSIC, UNESCO y Comisiones Obreras. No obstante, éstos, a su vez, se basan en las estadísticas del MECD.

La distribución por categoría es el resultado de varios cambios de normativas y formas de contratación. La última, a partir de 2001, cuando se creó la Agencia Nacional de Evaluación de la Calidad y Acreditación (ANECA) y reguló un proceso de acreditación en cuatro categorías. Dos de ellas, como funcionariado público: cátedra y titular de universidad, y otras dos con contrato laboral: ayudante doctor/a y contratado/a doctor/a. A estas últimas se unen la de profesorado ayudante, concebida para profesorado en proceso de formación, la cual no requiere el título de doctor/a. ${ }^{1}$

\footnotetext{
${ }^{1}$ En la categoría de otros/as funcionarios/as figura el profesorado titular y catedrático de Escuela Universitaria, creados con anterioridad a la última reforma y sin proceso de acreditación previa. El profesorado colaborador ocupa una figura antigua, extintita en nuevas contrataciones. El profesorado asociado son profesionales que tienen otra actividad principal. Los/as sustitutos/as, cubren temporalmente bajas coyunturales. En otros contratos laborales figura profesorado visitante, lector y emérito.
} 
Tabla 3. Distribución del personal docente e investigador de los centros propios de las universidades públicas españolas según categoría. Curso 2016-2017

\begin{tabular}{|c|c|c|c|c|c|c|}
\hline \multirow{2}{*}{ Categoría } & \multicolumn{2}{|c|}{ Total } & \multicolumn{2}{|c|}{ Mujeres } & \multicolumn{2}{|c|}{ Hombres } \\
\hline & Núm. & $\%$ & Núm. & $\%$ & Núm. & $\%$ \\
\hline Personal Funcionario & 43318 & 44.7 & 15463 & 39.0 & 27855 & 48.3 \\
\hline Cátedra de Universidad & 10017 & 10.3 & 2136 & 54.0 & 7881 & 13.7 \\
\hline Titular de Universidad & 28057 & 29.0 & 11298 & 28.8 & 16759 & 29.1 \\
\hline Otros & 5244 & 5.4 & 2029 & 2.0 & 3215 & 5.6 \\
\hline Personal Laboral & 53541 & 55.3 & 23778 & 60.6 & 29763 & 51.7 \\
\hline Contratado/a Doctor/a & 11347 & 11,7 & 5520 & 14.1 & 5827 & 10.1 \\
\hline Ayudante Doctor/a & 3223 & 3.3 & 1623 & 4.1 & 1600 & 2.8 \\
\hline Ayudante & 575 & 0.6 & 280 & 0.7 & 295 & 0.5 \\
\hline Profesorado colaborador & 2026 & 2.1 & 939 & 2.4 & 1087 & 1.9 \\
\hline Asociados/as & 31550 & 32.6 & 13125 & 33.4 & 18425 & 32.0 \\
\hline Profesorado sustituto & 2570 & 2.7 & 1394 & 3.6 & 1176 & 2.0 \\
\hline Otros & 2250 & 2.3 & 897 & 2.3 & 1353 & 2.3 \\
\hline Total & 96859 & 100.0 & 39241 & 100.0 & 57618 & 100.0 \\
\hline
\end{tabular}

Fuente: elaboración propia a partir del MECD (2018).

En esta estructura de clasificación, se establece el techo de cristal en el acceso a la categoría de funcionariado y/o en el acceso a la categoría de catedrático/a, y la forma de medirlo es doble. Por una parte, valorar el porcentaje de mujeres y hombres en la categoría más alta; $21.3 \%$ de catedráticas y $78.6 \%$ de catedráticos $(36 \%$ y $64 \%$, respectivamente, si se considera todo el funcionariado). La segunda forma es calcular el porcentaje sobre el total de cada sexo: algo menos de una de cada 18 mujeres, y algo menos de uno de cada 7 hombres en la cátedra ( 2 de 5 y 1 de 2, en funcionariado). En ambos casos, las diferencias tienen una magnitud importante y describen el techo de cristal en la cima de la carrera profesional académica.

\section{Los métodos de investigación utilizados}

De los documentos, 22 de los 46 documentos apor$\tan$ nuevas evidencias cuantitativas o cualitativas basadas en cinco técnicas: entrevistas, encuestas, grupos de discusión, historias de vida y observación directa. Ocho de las investigaciones utilizan más de una herramienta de recolección de información (tabla 4). El resto de estudios analizados se ocupan de la descripción del fenómeno a través de la revisión documental y/o estadística.

La entrevista se sitúa como el método de investigación empírico más utilizado, normalmente en un formato semiestructurado, con preguntas abiertas y un amplio margen de observaciones: desde tres (Guil et al., 2005) hasta 150 entrevistas (Puy, 2007). Suele estar 
dirigido al PDI, aunque puede ser extensivo al alumnado, al personal de administración y servicios (PAS) y a cargos de gestión. Excepcionalmente, se incluyeron entrevistas a especialistas en estudios de género (García de León, 2010). Cabe señalar que seis de estos trabajos sólo recabaron información de mujeres.

El segundo método más utilizado fue la encuesta. Se encuentra presente en nueve investigaciones y, en casi todos los estudios estuvieron dirigidas únicamente al PDI, mayoritariamente a ambos sexos. El tamaño de la muestra fue desde los 35 individuos (Matus-López y Gallego-Morón, 2015) hasta los 818 sujetos (Escolano, 2006), y los errores muestrales se situaron en casi todos los casos por encima del $5 \%$.

Una tercera técnica fue la de los grupos de discusión. Cuatro trabajos lo utilizaron. La mitad centrado en las mujeres PDI, pero otros dos incluyeron también varones (Tomás y Durán, 2009), así como PAS y alumnado (Martínez et al., 2011). La mayor muestra alcanzó a ocho grupos (Guil, 2005b), y la menor a un único grupo de 20 personas (Martínez et al., 2011).

Finalmente, dos estudios han utilizado como metodología la observación directa y uno de ellos la ha complementado con el análisis de historias de vida. La observación directa fue aplicada en dos formatos. El primero, de tipo formato libre y sin esquemas, centrado en observaciones participantes a las relaciones en clase entre PDI y alumnado, con un número no especificado de sujetos (Cinta y Velázquez, 2013). El otro formato fue sistémico categorial, centrado en la toma de decisiones llevadas a cabo en ocho reuniones, con casi dos centenares de participantes (Tomás y Durán, 2009).

Finalmente, aunque las historias de vida se constituyen como uno de los métodos descriptivos más enriquecedores y potentes, sólo se encuentra presente en un único trabajo, en el que no se detalla el número ni las características de los sujetos analizados. En resumen, las metodologías en este campo son variadas, con fortalezas y debilidades. La mayor fortaleza es que el número de estudios ha permitido construir un estado del arte en esta problemática, pudiendo evaluar las situaciones particulares de cada caso. Las principales debilidades, en cambio, son lo reducido del tamaño de las muestras, a nivel nacional, y la innovación en nuevas metodologías de investigación.

Tabla 4. Estudios según metodología, población y observaciones

\begin{tabular}{|c|c|}
\hline Autores/as & Metodología, población y número \\
\hline Antón (2005) & Encuesta a mujeres PDI $(n=200)$ \\
\hline Cinta y Velázquez (2013) & Observación directa e historias de vida a PDI y alumnado (n=n.d.) \\
\hline Durán e lon (2014) & Entrevistas a mujeres PDI $(n=7)$ \\
\hline Escolano (2006) & Entrevistas a mujeres PDI y Gestión ( $n=14)$; Encuesta a mujeres PDI ( $n=818$ ) \\
\hline García de León (2010) & Entrevistas a PDI y especialistas $(n=52)$ \\
\hline Guil (2005b) & Grupos de discusión a mujeres PDI (8 grupos de 6-8 personas) \\
\hline Guil et al. (2005) & $\begin{array}{l}\text { Entrevistas a personas en Gestión }(n=3) \text {; Encuesta a PDI }(n=240) \text { y PAS }(n=240) \text {; Grupos de discusión de PDI y } \\
\text { PAS ( } 6 \text { grupos de 6-8 personas) }\end{array}$ \\
\hline lon et al. (2013) & Entrevistas a PDI $(n=22)$ \\
\hline
\end{tabular}




\begin{tabular}{|c|c|}
\hline Lozano et al. (2014) & Entrevistas a mujeres PDI $(n=89)$ \\
\hline Martínez et al. (2011) & Grupos de discusión a PDI, PAS y Alumnado (1 grupo de 20 personas) \\
\hline Matus-López y Gallego-Morón (2015) & Encuesta a PDI $(n=35)$ \\
\hline Morales et al. (2010) & Entrevistas a informantes claves ( $\mathrm{n}=\mathrm{n} . \mathrm{d}$.) \\
\hline Pastor et al. (2010) & Encuesta a PDI $(n=330)$ \\
\hline Puy (2007) & Entrevistas y Encuestas a PDI, PAS y Alumnado $(n=150)$ \\
\hline Simelio y Rovetto (2008a, 2008b) & Entrevistas a mujeres PDI $(n=5)$ \\
\hline Tomás et al. $(2008,2010)$ & Entrevistas s mujeres PDI y en Gestión ( $n=18)$; Encuesta a PDI $(n=150)$ \\
\hline Tomás y Durán (2009) & Grupos de discusión (2 grupos) y Observación directa $(n=199)$ a PDI \\
\hline Tomás y Guillamón (2009) & Entrevistas a mujeres PDI y Gestión ( $n=18) ;$ Encuesta a PDI (N=150) \\
\hline Tomás et al. (2012) & Entrevistas a PDI y Gestión ( $n=22)$ \\
\hline Vázquez y Elston (2006) & Entrevistas a PDI $(\mathrm{n}=33)$ \\
\hline
\end{tabular}

\section{Las causas del techo de cristal en la academia}

La comprensión del techo de cristal y sus causas ha sido uno de los principales objetivos de la literatura existente. El 39\% de los estudios analizados lo han abordado de alguna manera (tabla 5). Las preguntas básicas de investigación detrás de estos estudios han sido ¿por qué la proporción de mujeres es menor en las categorías más altas de la academia que en las más bajas?, y ¿cuáles son las causas que afectan a las mujeres más que a los hombres para promocionar hasta la cúspide organizacional?

La forma de responder a estas preguntas tiene cuatro caminos. El primero es abordar la autopercepción que las propias académicas tienen de las carreras de las mujeres en la universidad así como sobre su situación personal. Otro es analizar las expectativas y opiniones que terceros tienen sobre las perspectivas de futuro en la participación y presencia de las mujeres en la academia. La tercera es interpretar desde fuera, desde un punto de vista externo, los discursos de las mujeres, particularmente de aquellas que han alcanzado la categoría de catedrática. Por último, un cuarto acercamiento es contrastar de manera empírica una hipótesis inicial para concluir a través de la evidencia empírica si se sostiene o no.

La mayor parte de los estudios no realizaron un proceso de causa-hipótesis-contrastación que fuera más allá de la descripción de las estadísticas oficiales. No obstante, ello no impide que citen o categoricen barreras. La tendencia general por parte de los/ as autores/as consiste en señalar de manera aislada dos o tres barreras que son coincidentes en algunos casos y, en otros no, con los resultados de las otras investigaciones. Cuando se han intentado agrupar estas causas, las categorías más utilizadas han sido la de barreras internas y externas (Tomás y Guillamón, 2009; Tomás et al., 2008, 2010), personales y/o familiares, organizacionales y sociales (Guil, 2005b) y barreras del sistema, del grupo y personales (Puy, 2007). En el presente trabajo, con el objetivo de abordar las causas señaladas en la literatura, se han agrupados estas en cuatro categorías: familiares, cognitivo-emocionales, organizacionales y sociales, relacionándolas con los estudios analizados (tabla 5). 
Tabla 5. Relación de autores/as según causas de las barreras del techo de cristal

\begin{tabular}{|c|c|c|}
\hline Barreras & Detalle & Estudios \\
\hline \multirow{3}{*}{ Familiares } & $\begin{array}{l}\text { Trabajo doméstico y de cuidados } \\
\text { Problemas de conciliación }\end{array}$ & $\begin{array}{l}\text { Antón (2005), Escolano (2006), García (2010), Guil (2005b), } \\
\text { Guil et al. (2005), lon et al. (2013), Martínez et al. (2011), Pastor } \\
\text { et al. (2010), Puy (2007), Sánchez et al. (2011), Simelio y Rovetto } \\
\text { (2008a, 2008b), Soleto y Oubiña (2005), Tomás y Guillamón } \\
\text { (2009), Tomás et al. (2008; 2010), Vázquez y Elston (2006) }\end{array}$ \\
\hline & Doble jornada laboral & García (2010), Guil (2005b), Guil et al. (2005) \\
\hline & Corresponsabilidad de hombres & Ion et al. (2013) \\
\hline \multirow{4}{*}{ Cognitivo-emocionales } & Autoestima y autoimagen & Guil (2005b), Tomás y Guillamón (2009) \\
\hline & Falta de confianza y miedo & Guil (2005b), Martínez et al. (2011), Tomás et al. $(2008,2010)$ \\
\hline & Sensación de culpa & Guil (2005b) \\
\hline & Auto-restricción & $\begin{array}{l}\text { Martínez et al. (2011), Tomás y Guillamón (2009), Tomás et al. } \\
(2008,2010)\end{array}$ \\
\hline \multirow{3}{*}{ Organizacionales } & Redes informales y Sistemas de cooptación & $\begin{array}{l}\text { De Filippo et al. (2009), Escolano (2006), García (2010), } \\
\text { Guil (2005b), Guil et al. (2005), Pastor et al. (2010), Tomás y } \\
\text { Guillamón (2009), Tomás et al. (2008, 2010), Vázquez y Elston } \\
\text { (2006) }\end{array}$ \\
\hline & Valoración de méritos & Guil et al. (2005), Sánchez et al. (2011), Tomás et al. $(2008,2010)$ \\
\hline & Falta de apoyo y financiación & Guil et al. (2005), Simelio y Rovetto (2008a, 2008b) \\
\hline \multirow{2}{*}{ Sociales } & Sociedad patriarcal & $\begin{array}{l}\text { Guil (2005b), Guil et al. (2005), lon et al. (2013), Martínez et al. } \\
\text { (2011), Tomás y Guillamón (2009), Tomás et al. }(2008,2010)\end{array}$ \\
\hline & Estereotipos de género & $\begin{array}{l}\text { García (2010), Guil (2005b), Martínez et al. (2011), Sánchez et } \\
\text { al. (2011), Tomás y Guillamón (2009), Tomás et al. }(2008,2010)\end{array}$ \\
\hline
\end{tabular}

\section{Barreras familiares}

Las dificultades de conciliación de la vida personal, laboral y social se constituye como la principal causa señalada en los estudios. Esta barrera consiste en que las mujeres deben hacerse cargo de una importante cantidad de trabajo doméstico y de cuidados no remunerados, que les resta tiempo y dedicación para alcanzar los méritos que le permitan progresar en la vida profesional. La diferencia con los hombres estaría en la inexistente corresponsabilidad de estos con este tipo de trabajos (Ion et al., 2013).
Así, las mujeres que ingresan al mercado laboral en casi cualquier empleo terminan asumiendo una doble jornada laboral: la de trabajo doméstico y de cuidados no remunerado, y la jornada laboral de mercado (García, 2010; Guil, 2005a; Guil et al., 2005). Mientras que los hombres tienen dedicación casi en exclusividad a esta última.

La consecuencia de esto, bajo una limitación natural de horas diarias, se traduce en que las mujeres desarrollan empleos con jornadas más cortas y/o no pueden dedicar el mismo tiempo que sus pares 
varones a la obtención de méritos profesionales. Una situación que caracteriza al mercado laboral en general y que es extensible a la carrera académica. Este menor tiempo de dedicación se traduce en menor participación en investigación, menos publicaciones y menos responsabilidades académicas, que pueden estar determinando el acceso a la cátedra.

Junto a este tipo de barreras, García (2010) señala como causante de la escasa representación de mujeres en los altos cargos y puestos de la pirámide profesional universitaria, la inexistencia de políticas públicas y medidas reales, concretas y efectivas de intervención que posibiliten la conciliación de la vida familiar, laboral y personal.

\section{Barreras cognitivas-emocionales}

Hombres y mujeres son educados y asisten a procesos de socialización distintos de género en función del sexo que se les asignó al nacer. En este proceso de socialización toman parte una serie de valores, estereotipos y prejuicios que son interiorizados y que construyen los pensamientos, sentimientos y valoraciones esperados de cada sexo.

Son varios/as los/as autores/as que señalan que los valores y sentimientos femeninos interiorizados por las mujeres les sitúan en condiciones de desventaja frente a los hombres en la carrera académica. Los estereotipos que de este proceso se derivan generan condiciones propicias para el desarrollo de problemas de autoestima y autoimagen (Tomás y Guillamón, 2009), lo que origina menos confianza en sus capacidades (Martínez et al., 2011), y muchas veces sensación de culpa al reducir la carga de trabajo doméstico y de cuidados (Guil, 2005b).

La mayoría de los estudios analizan estas cuestiones desde la perspectiva del resultado de procesos de socialización y educación. No obstante, también hay autores/as que lo hacen desde la perspectiva individual de las propias mujeres, como resultado de la acción social y la elección personal, produciéndose una auto-restricción para asumir cargos de responsabilidad. Se trata, en palabras de Tomás et al. (2008: 123), de una autorestricción que "ellas mismas se han impuesto y que las arraiga en la divergencia entre sus propios valores y los de la cultura universitaria dominante".

\section{Barreras organizacionales}

Gran parte de autores/as señalan entre los resultados de sus investigaciones la existencia de barreras dentro de la propia organización. Lo que se supone debe ser un sistema meritocrático, como es la universidad, se transforma en una red social-clientelar donde factores distintos al mérito académico toman un peso decisivo.

Las respuestas a las entrevistas y encuestas de estos trabajos confirman la existencia de redes masculinas de poder informales dentro de la universidad. La cercanía o distancia a estos círculos de poder favorece a unos/as candidatos/as y perjudica a otros/ as. Esta dinámica es lo que se denomina sistema de cooptación. Se trata de un mecanismo consistente en la elección de una persona candidata de modo subjetivo y directo, reduciendo la competencia o favoreciendo los méritos particulares de ésta.

Los estudios abordan otras dos causas, que en parte pueden estar ocasionadas por la anterior. La primera es que los méritos docentes y científicos de las mujeres académicas son valorados por debajo de méritos similares presentados por hombres en los concursos de promoción (Guil et al., 2005; Sánchez et al., 2011) o han sido sometidos a procesos de evaluación y promoción desarrollado de acuerdo con las normas masculinas, que discriminan a las mujeres (Tomás et al., 2008, 2010).

$\mathrm{El}$ segundo es la falta de apoyo y menor financiación económica a los equipos de investigación liderados por mujeres debido al sesgo en la valoración de méritos de estos. Ambos fenómenos cuentan con respaldo en la bibliografía internacional (Steinpreis et al., 1999; Van der Lee y Ellemers, 2015; Wennerás y Wold, 1997), aunque en el caso de los estudios 
nacionales revisados no se realizan contrastaciones empíricas de esta hipótesis.

\section{Barreras Sociales}

Las barreras sociales citadas en los estudios se refieren a la desventaja de las mujeres en la carrera académica que se derivan del carácter machista y patriarcal de la sociedad española en su conjunto (Guil, 2005b; Martínez et al., 2011; Sánchez et al., 2011; Tomás et al., 2008, 2010).

Múltiples son los estudios y encuestas sociales que muestran una discriminación estereotipada de las mujeres en la sociedad, basada en roles de género y transmitida a través de una socialización diferencial de género (García, 2010). Para algunos/as autores/ as, esta visión androcéntrica construye los perfiles de éxito y mérito profesional con patrones masculinos que ponen en desventaja a las mujeres. De acuerdo con los estudios analizados, la academia no está libre de este entono social y reproduce éstos en perjuicio de la promoción de las mujeres (Guil et al., 2005; Ion et al., 2013; Tomás y Guillamón, 2009).

\section{Conclusiones}

En el presente estudio se ha realizado una revisión sistemática de la literatura existente sobre el techo de cristal en las universidades españolas. Como ya mencionamos, éste supone un avance considerable en el conocimiento del fenómeno, en tanto en cuanto no nos consta la existencia de investigaciones previas que se ocupen en analizar, más allá de la revisión bibliográfica presente en todo artículo, de manera exhaustiva y sistemática el estado de la cuestión en España, desde una perspectiva internacional. Éste ofrece una panorámica de lo que se sabe y lo que se ignora, qué y cómo se ha investigado, y cuáles han sido los principales resultados. El estado de la cuestión nos presenta tres aspectos sobre los cuales reflexionar y proponer líneas de avance: 1) escasez de investigaciones empíricas, 2) estudios de casos aislados y 3) falta de consenso sobre las causas del fenómeno.
En primer lugar, la mitad de las investigaciones revisadas se basan de manera exclusiva en el análisis de fuentes estadísticas secundarias, lo que les impide contrastar muchas de las hipótesis. Aquellos que generan información nueva se basan principalmente en entrevistas y encuestas, y en mucha menor proporción en grupos de discusión, historias de vida y observación directa. Los resultados de estos estudios son más ricos pero adolecen, en su mayoría, de un escaso tamaño muestral y suelen estar sujetos a la interpretación de los/as autores/as. Algunos ejemplos interesantes en materia internacional, que han tratado de complementar la evidencia cualitativa con la cuantitativa, han sido los trabajos de Wennerás y Wold (1997) y el de Steinpreis et al. (1999). Estos/as autores/as contrastaron estadísticamente las evaluaciones hechas a currículos académicos de mujeres y hombres, determinando la significancia de la menor puntuación a mujeres, frente a un mismo mérito que los hombres.

En segundo lugar, las poblaciones estudiadas son restringidas. En ninguna ocasión representa el sistema universitario a nivel español. Así, los trabajos revisados se centran habitualmente en el estudio de casos aislados, como suele ser una o dos universidades de un país, o la profundización en ciertas ramas o campos de estudio (Matus-López y Gallego-Morón, 2015). Esto limita las comparativas entre comunidades autónomas y universidades. La evidencia muestra la universalidad del fenómeno en todos los ámbitos desarrollados e industrializados (Adler, 1986), por lo que los resultados particulares se asumen como extrapolables a nivel nacional, aunque no existe evidencia que lo demuestre.

En tercer lugar, una de las cuestiones más relevantes de este estudio es que a la hora de diagnosticar el techo de cristal en las universidades hay un elevado consenso de sus características. No obstante, en el análisis de las causas este consenso se diluye. Con base en las percepciones de las académicas, los/as propios/as autores/as realizan clasificaciones 
o identifican barreras de promoción, pero éstas tienen mucho en común con la discriminación de género en general y poco con la especificidad de las universidades. Así, existe cierta coincidencia en señalar los problemas de conciliación y una sociedad machista y estereotipada como barreras, pero menos en contrastar otras como las redes informales de poder y los sistemas de cooptación universitarios.

Los resultados coinciden con la revisión sistemática para otros países en términos de variedad metodológica y falta de evidencia empírica contrastable, sin que ello menoscabe la información cualitativa, y la carencia de universos nacionales (Jackson y O’Callaghan, 2009), lo que abre múltiples líneas de investigación en este campo. De este modo, y si se quiere seguir avanzando en pro de la igualdad de género en la academia, resulta imprescindible la realización de más estudios. Sin embargo, no basta con la proliferación de éstos, sino que es condición sine qua non que éstos cumplan una serie de requisitos:

1. Se debe recuperar la senda de los trabajos originales de García de León y García de Cortázar (2001), realizando estudios empíricos con enfoques metodológicos cualitativos, que permiten un análisis del fenómeno con mayor profundización a nivel nacional.

2. Se debe profundizar en el análisis de las barreras provenientes de la cultura organizacional, tales como la influencia de las redes informales de poder y los sesgos de género en la evaluación.

3. Además, éstos deben incidir no sólo en la identificación de las barreras y obstáculos que las mujeres deben superar, sino, además, en las medidas de intervención y políticas de actuación que desde la administración pública se deben aplicar.
Finalmente, cuestionamos la existencia de un único techo de cristal, considerado desde su concepción tradicional como una única barrera sólida que impide el acceso de las mujeres a los altos cargos. La literatura internacional confirma que existe una situación de discriminación de género continua desde los primeros niveles académicos, no sólo en los procesos de promoción a las categorías superiores, respondiendo a la existencia de un "laberinto de cristal" consistente en un entramada de barreras (Roldán et al., 2012). Sin embargo, los sujetos de estudio suelen estar conformados por profesorado perteneciente a las categorías superiores (Matus-López y Gallego-Morón, 2015). Por ello, nos preguntamos: ¿se presentan las barreras con la misma intensidad en todos los niveles académicos?, ¿son las mismas barreras en todas las categorías académicas?, ¿existen diferencias significativas según el grado de feminización de las ramas de conocimiento?, ¿han tenido efectos positivos las medidas y legislación en materia de igualdad de género aprobadas en la última década en España?

Una revisión como la realizada, además de poner de relieve los desafios a los que hacer frente en el estudio del fenómeno del techo de cristal en la academia, se constituye como diagnóstico previo para el diseño e implementación de medidas de intervención por parte de organismos públicos y de las propias universidades. La igualdad de oportunidades es un principio básico del desarrollo social. Además, la discriminación genera efectos negativos sobre la legitimidad social de las organizaciones y una pérdida de capacidad creativa y productiva de un país. Por ello, el acceso a la cúspide organizacional sólo debería estar condicionado por los méritos, más aún en un ámbito como el universitario. 


\section{Referencias}

Adler, Nancy J. (1986), "Women in management worldwide", International Studies of Management है Organization, vol. 16, núms. 3-4, pp. 3-32.

Arcos, Antonio, Antonio J. López-Montoya y María del Mar Rueda (2013), "Is it real gender equality in university?", ponencia presentada en el 7th International Technology, Education and Development Conference, Valencia, 4-6 marzo.

Antón, Susana (2005), Informe: Académicas en la Universidad de Alicante, Alicante, Universidad de Alicante.

Barberá, Ester, Amparo Ramos, Maite Sarrió y Carlos Candela (2001), "Más allá del techo de cristal. Diversidad de género", Revista del Ministerio de Trabajo y Asuntos Sociales, núm. 40, pp. 55-68.

Blahopoulou, Joana, Esperanza Bosch y Victoria Ferrer (2011), "Segregación horizontal y vertical en el colectivo del personal docente e investigador de la universidad. ¿Evolución o estancamiento?”, en Logros y retos: Actas del III Congreso Universitario Nacional Investigación y Género, Sevilla, Universidad de Sevilla, pp. 146-167.

Bozzon, Rosella, Annalisa Murgia, Barbara Poggio y Elisa Rapetti (2017), "Worklife interferences in the early stages of accademic careers: the case of precarious researchers in Italy", European Educational Research fournal, vol. 16 núm. 2-3, pp. 332-351.

Cáceres, María Pilar, Antonio Sachicola y María Angustias Hinojo (2015), "Análisis del liderazgo femenino y poder académico en el contexto universitario español", European Scientific Fournal, vol. 11, núm. 2, pp. 296-313.

Catalyst (2017), Quick take: women in academia, disponible en <https://www.catalyst.org/research/women-inacademia/> [Consulta: mayo de 2019].

Cinta, Elisabeth y María Isabel Velázquez (2013), "Desigualdades de género en las universidades de Sevilla. Feminización y masculinización en las aulas y los departamentos: un estudio comparativo entre trabajo social e ingeniería aeroespacial", Documentos de trabajo social: Revista de trabajo y acción social, núm. 53, pp. 43-59.
Conferencia de Rectores de las Universidades Españolas (CRUE) (2018), Universidades, recuperado de <http://www.crue.org/Universidades/SitePages/ universidades.aspx> [Consulta: mayo 2018].

De Filippo, Daniela, Elías Sanz e Isabel Gómez (2009), "Movilidad científica y género: estudio del profesorado de una universidad española", Revista Mexicana de Sociología, vol. 71, núm. 2, pp. 351-386.

Díaz, Francisco y Mercedes Cuevas (2015), "Género y liderazgo en la universidad española. Un estudio sobre la brecha de género en la gestión universitaria", Archivos Analíticos de Políticas Educativas, vol. 23, núm. 106, pp 1-26.

Durán, María del Mar y Georgeta Ion (2014), "Investigadoras con éxito en la universidad... ¿Cómo lo han logrado?”, Educación XX1, vol. 17, núm. 1, pp. 39-58.

Escolano, Esther (2006), "Discriminación en un medio meritocrático: las profesoras en la universidad española", Revista Mexicana de Sociología, núm. 2, pp. 231-263.

Eurofound (2016), The gender employment gap: Challenges and solutions, Luxembourg, Publications Office of the European Union.

European Commission (2019), She Figures 2018, Luxembourg, Publications Office of the European Union.

Fernández, Concepción (1989), "La mujer en la universidad española. Docencia, investigación y poder. Datos y aspectos cualitativos", Revista de Educación, núm. 290, pp. 161-171.

Flecha, Consuelo(2010), "Sin pedir permiso. Universitarias y funcionarias en 1910", Revista Crítica, núm. 969, pp. 66-69.

García, Antonia (2010), "Viejos retos y nuevas realidades de las mujeres en la educación superior", Revista de Antropología Experimental, núm. 10, pp. 29-46.

García de León, María Antonia (dir.) (2010), Las científicas sociales en España: una investigación acción. Año 2005-Año 2007, Madrid, Universidad Complutense de Madrid. 
García de León, María Antonia y María Luisa García de Cortázar (eds.) (2001), Las académicas. Profesorado universitario y género, Madrid, Instituto de la Mujer.

García de León, María Antonia y María Luisa García de Cortázar (1997), Mujeres en minoría: una investigación sociológica sobre las catedráticas de universidad en España, Madrid, Centro de Investigaciones Sociológicas.

Gewinner, Irina (2016), "Gender inequality in Russian Academia: dynamics, insights, and explanations", Investigaciones feministas, vol. 7, núm. 2, pp. 115-137.

Guil, Ana (2008), "Mujeres y ciencia: techos de cristal", Eccos Revista Científica, vol. 10, núm. 1, pp. 213-232.

Guil, Ana (2007), "Docentes e investigadoras en las universidades españolas: visibilizando techos de cristal", Revista de Investigación Educativa, vol. 25, núm. 1, pp. 111-132.

Guil, Ana (2005a), "Mujeres, universidad y cambio social: tejiendo redes", ponencia presentada en las I fornadas de Sociología: El cambio social en España. Visiones y retos de futuro, Sevilla, 15-16 junio.

Guil, Ana (2005b), Techos de cristal en la Universidad de Sevilla, Madrid, Ministerio de Trabajo y Asuntos Sociales, Instituto de la Mujer.

Guil, Ana, Ana Solano y Manuela Álvarez (2005), La Situación de las mujeres en las universidades públicas andaluzas, Sevilla, Consejo Económico y Social de la Junta de Andalucía.

Guil, Ana y Raquel Vila (2006), "Visibilizando diferencias entre áreas de conocimiento", ponencia presentada en el VI Congreso Iberoamericano de Ciencia, Tecnología y Género, Zaragoza, 10-15 septiembre.

Instituto Nacional de Estadística (INE) (1999), Estadística de la Enseñanza Universitaria en España, Curso 1998-1999, recuperado de <http://www.ine.es/dyngs/INEbase/ es/operacion.htm?c=Estadistica_C\&cid $=1254736$ $176744 \&$ menu $=$ resultados\&idp $=1254735573113>$ [Consulta: mayo de 2018].

INE (2016), Encuesta de Población Activa, Período 1976-2016, recuperado de <http://www.ine.es/dyngs/INEbase/
es/operacion.htm?c=Estadistica_C\&cid $=1254736$ $176918 \&$ menu $=$ resultados\&idp $=1254735976595>$ [Consulta: mayo de 2018].

Ion, Georgeta, María del Mar Durán y María Dolors Bernabeu (2013), "El profesorado y su percepción sobre la igualdad de género en la universidad", Revista Complutense de Educación, vol. 24, núm. 1, pp. 123-140.

Jackson, Jerlando L. y Elisabeth M. O'Callaghan (2009), "What do we know about glass ceiling effects? A taxonomy and critical review to inform higher education research", Research in Higher Education, vol. 50, núm. 5, pp. 460-482.

López, Jesús Manuel, María del Rocío Martínez y María del Carmen Díaz (2014), "Diferencias de género en las universidades españolas y en sus órganos gerenciales", Revista Venezolana de Gerencia, vol. 19, núm. 66, pp. 250-266.

Lorenzo, Manuel, Tomás Sola y María Pilar Cáceres (2007), "El liderazgo femenino en los cargos directivos: un estudio longitudinal en la Universidad de Granada (1990-2005)", Educación y Valores, vol. 10, núm. 2, pp. 177-194.

Lozano, Inés y Marco Jesús Iglesias (2014), "La presencia de las académicas a lo largo de la historia en la educación superior española", fournal for Educators, Teachers and Trainers, vol. 5, núm. 3, pp. 204-216.

Lozano, Inés, Marco Jesús Iglesias y María Ángeles Martínez (2014), "Las oportunidades de las académicas en el desarrollo profesional docente universitario: un estudio cualitativo", Educación XX1, vol. 17, núm. 1, pp. 159-182.

Martínez, María del Rocío, Jesús Manuel López y María del Carmen Díaz (2010), "Estudio de género en los órganos decisorios de la universidad", en Investigaciones multidisciplinares en género: Actas del II congreso universitario nacional Investigación y Género, Sevilla, Universidad de Sevilla, pp. 681-691.

Martínez, María del Rocío, María Ángeles Serrano, María del Carmen Díaz y Jesús Manuel López (2011), "Identificación de las principales dificultades que obstaculizan el acceso a los órganos de gobierno en las 
universidades españolas", en Logros y retos: Actas del III congreso universitario nacional Investigación y Género, Sevilla, Universidad de Sevilla, pp. 1239-1257.

Matus-López, Mauricio y Nazareth Gallego-Morón (2015), "Techo de cristal en la universidad. Si no lo veo no lo creo", Revista Complutense de Educación, vol. 26, núm. 3, pp. 611-626.

Ministerio de Educación, Cultura y Deporte (MECD) (2018), Estadística de personal de las universidades (EPU), Curso 2016-2017, recuperado de <http://www.mecd. gob.es/servicios-al-ciudadano-mecd/estadisticas/ educacion/universitaria.html> [Consulta: mayo de 2018].

Méndez, Raquel y Mauricio Rojas (2014), "El género: una variable invisible pero sustantiva en la educación superior", Sophia, vol. 10, núm 1, pp. 74-82.

Mingorance, Pilar, Carlos Calderón y Araceli Estebaranz (2004), Andaluzas de hoy. Mujeres que abren caminos en la educación y en la ciencia, Córdoba, Ministerio de Trabajo y Asuntos Sociales.

Montané, Alejandra y Maria Eulina Pessoa (2012), "Diálogo sobre género: justicia, equidad y políticas de igualdad en educación superior (Brasil y España)", Revista Lusófona de Educação, núm. 21, pp. 97-120.

Morales, María Jesús, María José Luna y Ana Isabel Esteban (2010), "Diagnóstico de paridad en la universidad: análisis a través de indicadores", Revista de Universidad y Sociedad del Conocimiento, vol. 7, núm. 2, pp. $1-14$.

Morrison, Ann M., Randall P. White y Ellen Van Velsor (1987), Breaking the glass ceiling: can women reach the top of America's largest corporations?, Nueva York, AddisonWesley.

National Center for Education Statistics (NCES) (2017), Characteristics of Postsecondary Faculty, disponible en $<$ https://nces.ed.gov/programs/coe/indicator_csc. asp> [Consulta: mayo de 2019].

O’Connor, Pat et al. (2017), "Micro-political practices in higher education: a challenge to excellence as a rationalising myth?", Critical Studies in Education, pp. 1-17.

Pastor, Inma, Ángel Belzunegui, Blanca Moreno y Carmen Mañas (2010), "La igualtat d'oportunitats a la universitat: les percepcions del PDI", Papers: Revista de Sociología, vol. 95, núm. 2, pp. 457-481.

Pérez, Eulalia (dir.) et al. (2005), Mujer y ciencia. La situación de las mujeres investigadoras en el sistema español de ciencia y tecnología, Madrid, Ministerio de Educación y Ciencia, Fundación Española para la Ciencia y Tecnología.

Peterson, Pamela L. (2016), "Women as transformative leaders in higher education: an interpretive multi-case study", Doctoral dissertation, United States, University of St. Thomas.

Poy, Raquel (2009), "Mujeres y universitarias: historia de un desencuentro en la universidad española contemporánea", Cuestiones de género: de la igualdad y la diferencia, núm. 4, pp. 357-381.

Probert, Belinda (2005), “'I just couldn't fit it in': gender and unequal outcomes in academic careers", Gender, Work ङ Organization, vol. 12, núm. 1, pp. 50-72.

Puy, Ana (dir.) (2007), Barreras a la promoción de las mujeres a puestos de alto status en la universidad, Madrid, Ministerio de Igualdad, Instituto de la Mujer.

Rebolledo, Loreto y María Paulina Espinoza (2016), "Género, universidad e investigación. Una tríada compleja", Anales de la Universidad de Chile, vol. 11, pp. 155-171.

Rodríguez, Adelina (2006), "Educación superior y segregación de género: órganos unipersonales de la Universidad de León”, Cuestiones de género: de la igualdady la diferencia, núm. 1, pp. 137-153.

Roldán, Elena, Begoña Leyra y Leticia Contreras (2012), "Segregación laboral y techo de cristal en Trabajo Social: análisis del caso español”, Portularia: Revista de Trabajo Social, vol. 12, núm. 2, pp. 243-56.

Sales, Laura (2009), "El techo de cristal de las investigadoras: la ciencia incompleta", Enfermería Clínica, vol. 19, núm. 1, pp. 1-3.

Sánchez, Inés, Sara de la Rica y Juan José Dolado (2011), Libro Blanco sobre la situación de las mujeres en la ciencia española, Madrid, Unidad de Mujeres y Ciencia, Ministerio de Ciencia e Innovación.

Simelio, Nuria y Florencia Rovetto (2008a), "La mujer joven en la universidad ¿una doble discriminación?”, 
en Actas del I Congreso Internacional sobre sesgo de género y desigualdades en la evaluación de la calidad académica, Barcelona, Universitat Autónoma de Barcelona, pp. 291-305.

Simelio, Nuria y Florencia Rovetto (2008b), "La situación de las profesoras jóvenes en la Facultad de Ciencias de la Comunicación de la UAB", Zer: Revista de Estudios de Comunicación, núm. 25, pp. 287-307.

Soleto, Helena y Sabela Oubiña (2005), "La conciliación de la vida familiar y la docencia en la universidad: concreto estudio de la situación en la Universidad Carlos III", en Certamen de Ensayo sobre Igualdad y Género 2005 del Observatorio de Género, Madrid, Universidad Carlos III, pp. 2-24.

Steinpreis, Rhea E., Katie A. Anders y Dawn Ritzke (1999), "The impact of gender on the review of the curricula vitae of job applicants and tenure candidates: A national empirical study", Sex Roles, vol. 41, núm. 7, pp. 509-528.

Tomás, Marina, Diego Castro y María del Mar Durán (2012), "Aproximación a un modelo de análisis de la visibilidad en la universidad desde la perspectiva de género", Bordón, vol. 64, núm. 1, pp. 141-155.

Tomás, Marina y María del Mar Durán (2009), “El género y la participación en algunos órganos de gobierno en la universidad", Revista Complutense de Educación, vol. 20, núm. 1, pp. 151-163.

Tomás, Marina, María del Mar Durán, Cristina Guillamón y José Manuel Lavié (2010), "Women in academic administration at the university", Educational Management Administration and Leadership, vol. 38, núm. 4, pp. 487-498.

Tomás, Marina, María del Mar Durán, Cristina Guillamón y José Manuel Lavié (2008), "Profesoras universitarias y cargos de gestión", Contextos educativos:
Revista de educación, núm. 11, pp. 113-130.

Tomás, Marina y Cristina Guillamón (2009), "Las barreras y los obstáculos en el acceso de las profesoras universitarias a los cargos de gestión académica", Revista de Educación, núm. 350, pp. 253-275.

Torres, Obdulia y Bernadette Pau (2012), "Techo de cristal y suelo pegajoso: la situación de la mujer en los sistemas alemán y español de ciencia y tecnología", CTS: Revista Iberoamericana de Ciencia, Tecnología y Sociedad, vol. 6, núm. 18, pp. 35-59.

UNESCO Institute for Statistics (UIS) (2019), UNESCO eAtlas of Gender Inequality in Education, recuperado de <https:/ / tellmaps.com/uis/gender/> [Consulta: mayo de 2019].

United Nations (2015), The World's Women 2015: Trends and Statistics, Nueva York, Department of Economic and Social Affairs.

Van der Lee, Romi y Naomi Ellemers (2015), "Gender contributes to personal research funding success in The Netherlands", Proceedings of the National Academy of Sciences, vol. 112, núm. 40, pp. 12349-12353.

Vázquez, Susana y Mary Ann Elston (2006), “Gender and academia career trajectories in Spain. From gendered passion to consecration in a sistema endogámico?", Employee Relations, vol. 28, núm. 6, pp. 588-603.

Vega, Ana y José Diego Santos (2010), "La mujer en la Universidad de La Laguna", Qurriculum: Revista de teoría, investigación y práctica educativa, núm. 23, pp. 131-154.

Wennerás, Christine y Agnes Wold (1997), "Nepotism and sexism in peer-review", Nature, vol. 387, núm. 6631, pp. 341-343.

Wesarat, Phathara-on y Jaya Mathew (2017), "Theoretical framework of glass ceiling: a case of India's women academic leaders", Paradigm, vol. 21, núm 1, pp. 21-30.

Cómo citar este artículo:

Gallego-Morón, Nazareth, Mauricio A. Matus-López y Lina Gálvez-Muñoz (2020), "Revisión sistemática de la literatura sobre el fenómeno del techo de cristal en las universidades españolas", Revista Iberoamericana de Educación Superior (RIES), vol. XI, núm. 31, pp. 130-149, DOI: https://doi.org/10.22201/iisue.20072872e.2020.31.710 [Consulta: fecha de última consulta]. 